Check for updates

Cite this: RSC Adv., 2019, 9, 2865

Received 10th August 2018

Accepted 23rd December 2018

DOI: $10.1039 / \mathrm{c} 8 \mathrm{ra06717a}$

rsc.li/rsc-advances

\section{One step DNA amplification of mammalian cells in picoliter microwell arrays $\dagger$}

\author{
Wenwen Liu, (D) Zhao Li, Yuanjie Liu, Qingquan Wei, Yong Liu, Lufeng Ren, \\ Chenyu Wang (D) and Yude Yu*
}

We developed a strategy for direct DNA amplification of single cells on a PEG-modified silica chip with 30600 picoliter-sized microwells. HPV-positive cells in heterogeneous populations were successfully detected with high accuracy sensitivity as high as single copy.
Sensitive and specific detection of nucleic acids from rare but biologically important individual cells can bring in-depth understanding of cell differentiation and disease occurrence; for example, stem cells and circulating tumour cells in cancer patients. ${ }^{\mathbf{1 - 4}}$ As the most powerful and basic technologies to analyse nucleic acids, polymerase chain reaction (PCR) produces millions of copies of DNA molecules starting from a single template DNA. In PCR of cell populations, cell lysis and specific enrichment procedures need to be highly efficient and compatible with downstream analysis, which may lose rare DNA and cannot reveal cell heterogeneity. Methods for isolating and PCR of single cells are essential to obtain more meaningful information.

Several research groups have reported techniques for gene analysis using a small amount of cells, even a single cell..$^{5-9}$ Flow cytometry may be the most effective way for isolating large numbers of individual cells, but gene analysis of these sorted cells is still conducted in vials, with microliter volumes and low throughput. Analytical tools and methods for high throughput gene analysis based on microfluidic technology have emerged in recent years, ${ }^{\mathbf{1 0 , 1 1}}$ including micro wells and micro droplets. Fusion and division of droplets are needed to add reagent and reduce lysate-induced inhibition effects, but they destroy the integrity of $\mathrm{DNA}^{\mathbf{1 2}}$ and the accuracy of detection. Meanwhile, sample transfers between generation and operation of droplets, DNA amplification, and fluorescent detection also induces high risk of sample loss. Last but not least, complicated fabrication and operation processes, as well as high commercial costs of these microfluidic chips, are not affordable by most biological labs. One-step PCR of cells in micro wells without sample transfer is an effective and low cost method to ensure the integrity of cell DNA. However, due to lysate-mediated inhibition, reducing reaction volume to

State Key Laboratory on Integrated Optoelectronics, Institute of Semiconductors, Chinese Academy of Sciences, P. O. Box 912, Beijing, 100083, China. E-mail: yudeyu@semi.ac.cn

$\dagger$ Electronic supplementary information (ESI) available. See DOI: $10.1039 / \mathrm{c} 8 \mathrm{ra} 06717 \mathrm{a}$ picoliters for high throughput analysis of cells is still a challenge today.

In this study, we present a strategy for one-step DNA amplification of a single cell on a PEG-modified silica chip with 30600 picoliter-sized microwells. We applied this strategy to detect HPV 16 E6 positive cells with existence of negative cells as background, demonstrating its feasibility for medical diagnosis and related applications. Compared with flow cytometry or microfluidic chips, this strategy can robustly detect a single copy of nucleic acids from single cells within 2.5 hours by an integrated operation. It is dramatically simpler and cheaper than previously reported methods, thus more accessible by most biology labs.

The workflow of our approach, shown as Fig. 1, illustrates the size-optimized silica chip is $2.2 \times 2.2 \mathrm{~cm}^{2}$ and has 30600 microwells and is $100 \mu \mathrm{m}$ in diameter, $40 \mu \mathrm{m}$ in depth, and 314 pL in volume (Fig. 1A). ${ }^{13}$ To avoid air bubbles and protein

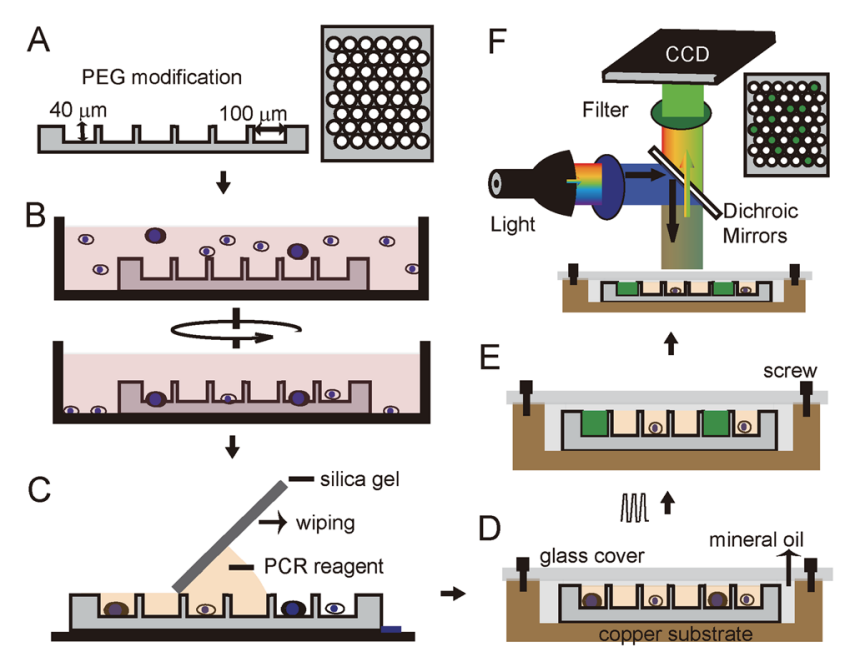

Fig. 1 Working flow of one-step PCR for single cells. (A) Silica chip with microwells. (B) Loading of cells. (C) Division of PCR reagent. (D) Assembled heating chamber. (E) Heating chamber containing target cells generate fluorescent signal during PCR. (F) The fluorescent signal can be detected by a wide-field microscope. 
adsorption during sample loading, surfaces of the chip were modified by methoxy-PEG-silane (2-[methoxy(polyethyleneoxy) propyl]-trimethoxysilane). Our method for fabricating the heating chamber is as follows: we first dried the silica chip and put it on the bottom of a culture dish. A pre-treated cell suspension with optimized density was added to the culture dish and then subjected to centrifugation (Fig. 1B). After cells were loaded into the microwells, the chip was washed with water and dried again. Then, PCR reagent was added on one side of the chip and a piece of silica gel was used to wipe the PCR reagent to another side (Fig. 1C). Next, the chip was sandwiched in a copper-glass chamber, which was filled with mineral oil and fixed by screws (Fig. 1D and S1†). Finally, this heating chamber was put on top of the heating module of a thermal cycler for PCR assay. TaqMan probes specific to target sequence produce a detectable signal when the positive cell is present and this PCR signal can be detected either during or at the end point of the assay by a fluorescence microscope (Fig. 1E and F).

As the simplest and most accessible method, centrifugation was used to load cells into microwells. According to single cell trapping analysis that was previously demonstrated, the number of wells containing single cells climbs and then declines with increasing cell density. ${ }^{14}$ To obtain the maximum amount of single cells, we optimized density of the cell suspension for loading by increasing cell density gradually in a relatively small range and recording the number of cells in the microwell. The correlation between cell density and cell numbers in microwells is shown as Fig. 2. As expected, both the numbers of wells containing single cells and multi-cells increased with increased loading density, but the percentage of single cells decreased gradually. To balance these two aspects, we chose a loading density between $40000-60000$ cells per $\mathrm{mL}$. At this constant parameter, we obtained 8000 wells with cells, and more than $80 \%$ of these wells contained a single cell.

We used the same chip for cell loading and PCR reagent division to ensure the integrity of the cell's nucleic acids. There are several hindrances to fulfilling this strategy. The first one is to reduce the phosphate-buffered saline (PBS) normally used to suspend live cells during cell loading. Although the microwell is picoliter-sized, PBS salt precipitated when drying the chip after cell loading and was resolved during the PCR reagent division. The pretreatment of a cell sample we used was to eliminate PBS from cell suspension through fixing cells by paraformaldehyde (PFA) and re-suspending them in deionized water. Therefore, both PBS and PFA were eliminated. Our fixation process prevented cells from bursting during buffer replacement and also inactivated enzymes that inhibit a PCR reaction. Our results demonstrated that PBS elimination increased the PCR efficiency dramatically (Fig. S2 $\dagger$ ). On the other hand, suspending cells in water can keep the surface of a chip clean enough for PCR reagent division. Otherwise, air bubbles will generate when wiping the PCR reagent, become larger, and move during the thermal cycle, bringing strong fluorescent background finally (Fig. S2†).

The lysate-mediated inhibition effect increased dramatically when the reaction volume decreased to picoliter-scale, not only
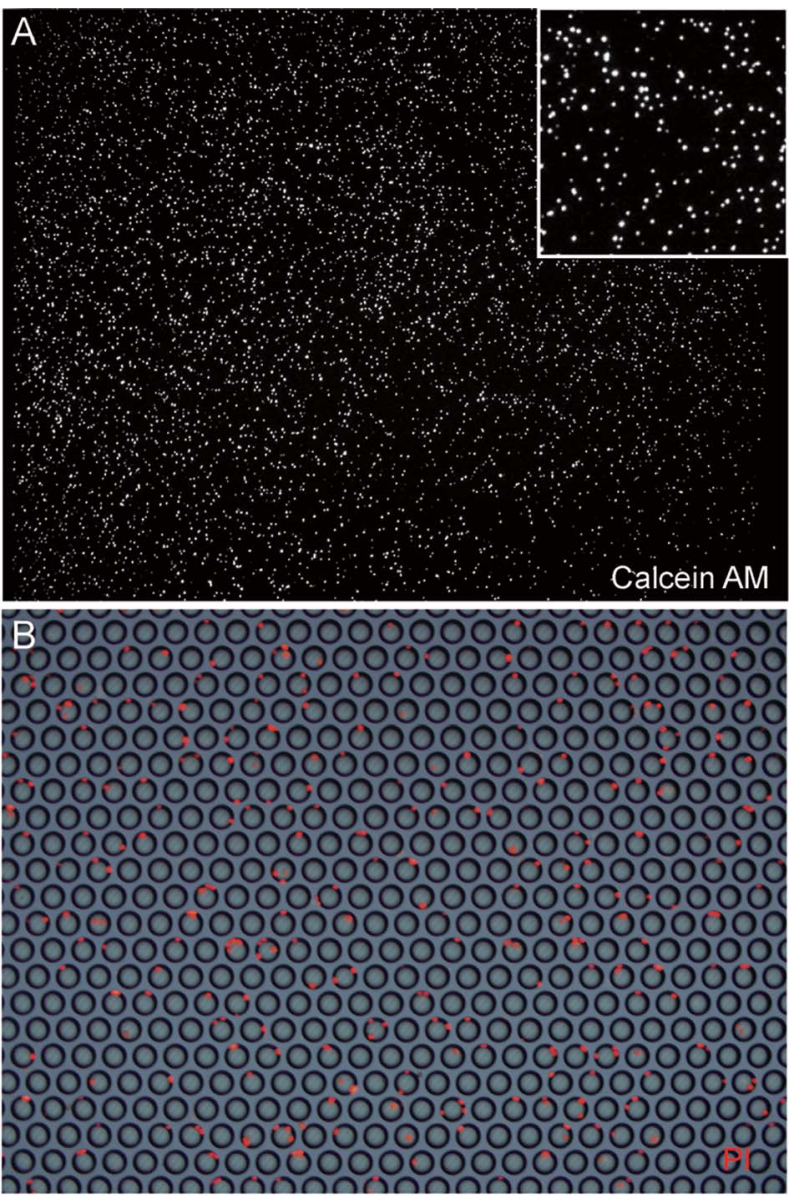

C

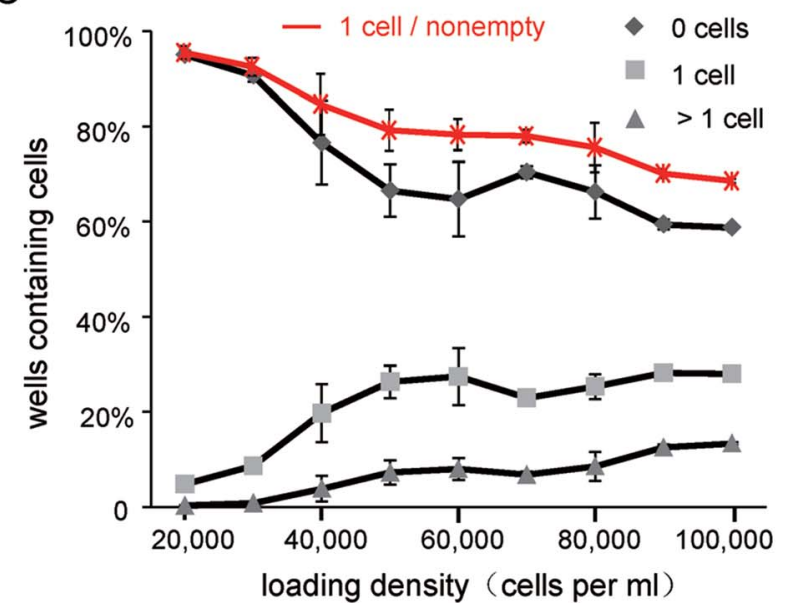

Fig. 2 Cell loading analysis. (A) Chip after loading live cells stained by calcein AM. (B) Bright field and fluorescent images of higher magnification images show the PI labeled cells in a microwell. (C) Relation between the loading density of cell suspension and the cell distribution on a chip. Percentage of single cells among all nonempty wells (red line) decreased with increased cell loading density.

because of the remaining lysis reagent itself, but also due to the high concentration of cell lysate. ${ }^{15-18}$ According to reported works that BSA could be a mild reagent for direct cell lysis, ${ }^{19,20}$ another effort we made to fulfill this strategy was the introduction of bovine serum albumin (BSA) to PCR reagent. 

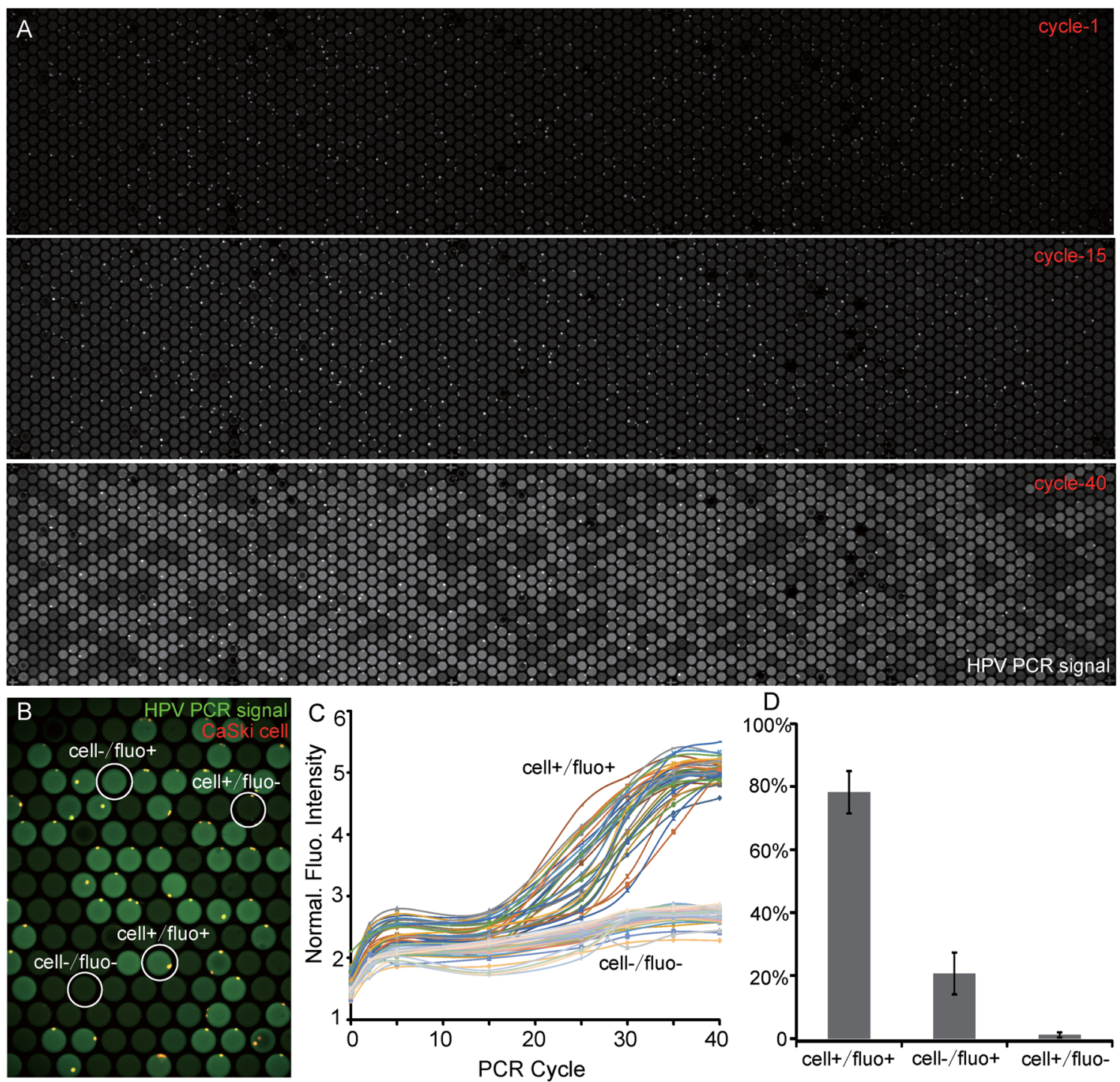

Fig. 3 One-step PCR of single CaSki cells for detecting HPV DNA. (A) Images of the same chip during PCR on cycles 1, 15, and 40. Bright spots in cycle-1 and cycle-15 are nonspecific fluorescence of cells, and the bright wells in cycle 40 are PCR signals. (B) Definition of microwells. Cell+/ fluo+ is a microwell with both cell and PCR signals; cell-/fluo+ is a microwell with a PCR signal but without cells; cell+/fluo- is a microwell that contains cells, but no PCR signal; cell-/fluo- is empty background. (C) Fluorescence intensity curve illustrating the normal amplification of cells. (D) Statistical analysis of the accuracy of this strategy. The diameter of a well is $100 \mu \mathrm{m}$.

Compared with traditional PCR in a vial, PCR at picoliter scale has higher surface to volume ratio; thus, we needed a high enough concentration of BSA to lysis cells and prevent interfaceadsorption of DNA polymerase. Unfortunately, we also found that background noises became stronger as BSA concentration increased. Considering these two contradictory aspects, we finally optimized the BSA concentration at $25 \mathrm{mg} \mathrm{mL}{ }^{-1}$, which is a very high concentration compared with $1 \mathrm{mg} \mathrm{mL} \mathrm{m}^{-1}$ in traditional PCR carried out in vials and at which background noises are acceptable for detection.

Human papilloma virus (HPV) induced cervical cancer was used as a model system to demonstrate the feasibility of this method. It is reported that HPV 16 E6 gene mediates the oncogenic property of HPV and over $99 \%$ of all cervical cancers are high-risk HPV-positive. ${ }^{21,22}$ The detection of HPV positive cells can be used as a primary diagnosis. We first verified the
PCR reagent and PEG-modified chip worked well by cell lysate (Fig. S3A and B $\dagger$ ). Next, we detected the HPV DNA in CaSki cells (HPV+, 600 copies) and human Alu elements (Fig. S4†), which are the most abundant transposable elements dispersed throughout the human genome. ${ }^{23,24}$ For both of these cases, we obtained the same specific PCR signal after 25 cycles (Fig. 3A and $\mathrm{S} 4 \dagger)$. To exclude false signals, CaSki cells shown in Fig. 3A were not fluorescent labelled. Nevertheless, a cell itself generated a fluorescent signal immediately after the initial denaturation of PCR and this non-specific fluorescence changed very little during the thermal cycle. The only reason may be that cells adsorbed some TaqMan probes that were destroyed during denaturation. On the contrary, we took advantage of this property to identify the cell itself from a PCR signal in the following experiments when the cells were not initially stained. 


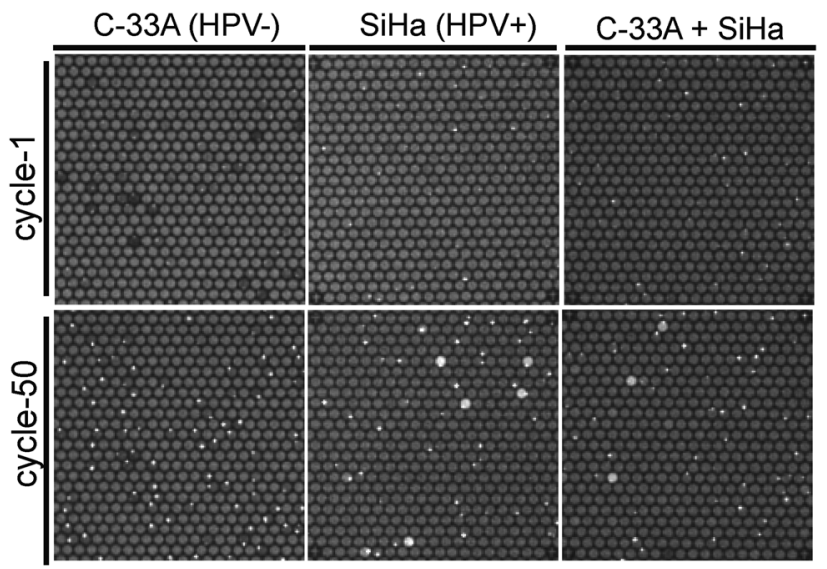

Fig. 4 Detection of HPV 16 E6 in negative cells, positive cells, and heterogeneous populations. No PCR signals were generated in C-33A cell after 50 cycles. Single copy of HPV16 could be detected in both $\mathrm{SiHa}$ cells and the cell mixtures.

We also stained cells by PI before loading them into microwells to distinguish cells from the PCR signals more clearly. We defined the relationship of cell-PCR fluorescence by a higher magnification shown as Fig. 3B and took images after each 5 cycles. The fluorescence of 35 cell+/fluo + and 35 cell-/fluowere analyzed. The curve of cell+/fluo+ shows an "S" shape which indicates a normal amplification and also a significant difference from the cell-/fluo- (empty well, Fig. 3C). On the other hand, it is easy to find that all the cell-/fluo+ (false positive signal) are near cell $+/$ fluo $^{+}$(Fig. 3B), which indicated the possibility of leakage from a neighboring positive well. As these false positive signals are always together with positive signals, and statistics suggest that the ratio of cell+/fluo- is $1.1 \%$ (Fig. 3D), we can conclude that once a positive cell appears, it could be detected with an accuracy as high as $98.9 \%$ (sum of cell+/fluo+ and cell-/fluo+).

In order to confirm the utility of this assay to identify specific cells in heterogeneous populations, we detected HPV 16 gene in $\mathrm{SiHa}$ cells (HPV+) based on TaqMan PCR assays with existence of C-33a cell (HPV-) as background, which were not infected by HPV. Unlike the large number of HPV in CaSki and Alu element in human cells, each SiHa cells contain 1-2 copies of HPV 16 DNA only on average. ${ }^{25-27}$ Consistent with what we predicted, HPV-negative cells C-33A showed no PCR signals after 50 thermal cycles (Fig. 4). We found that SiHa cells, but not all of them ( $23 \%$ on average), generated positive PCR signals, which is different from what we originally thought (Fig. 4). We also mixed SiHa cells and C-33A cells to decline the positive cell content and the positive signals decreased continually. These findings demonstrated our strategy successfully detected a single copy of target DNA from single cells among heterogeneous populations.

There are several possibilities for the contradiction on HPV 16's copies in a SiHa cell. First, the reported number of 1-2 copies on average may be too low to detect in the picoliter-sized wells shown in our strategy, especially with a high concentration of protein as background. The second is that not every SiHa cell really has HPV 16 DNA because the reported data is an average number. More data are needed to give a deeper explanation through single cell sequencing or digital PCR of single SiHa cells.

\section{Conclusions}

In conclusion, we have established a novel strategy for the detection of specific cells in picoliter-sized reaction units with high throughput and efficiency. The cell pretreatment described in this work eliminates or inactivates most inhibitors, allowing the PCR reaction to be performed in picoliter-sized microwells. Using an improved PCR reagent, no specific cell lysis was needed for successful identification of a single DNA copy from single HPV positive cells in a microwell. Compared with previous reported single cell detection on a chip, simplification of the device and operation, combined with a commercial PCR instrument, and the high sensitivity of single copy detection in this work, enables the strategy to be dramatically more accessible by biologists. We also believe that this strategy of loading and direct PCR of single cells can be useful in a broad array of applications, especially single cell sequencing.

\section{Conflicts of interest}

There are no conflicts to declare.

\section{Acknowledgements}

This work was supported by grants from the Instrument Developing Project of the Chinese Academy of Sciences (Grant No. YZ201301) and the National Natural Science Foundation of China (Grant No. 61334008, 21804126).

\section{Notes and references}

1 M. Etzrodt, M. Endele and T. Schroeder, Cell Stem Cell, 2014, 15, 546-558.

2 A. Saadatpour, S. J. Lai, G. J. Guo and G. C. Yuan, Trends Genet., 2015, 31, 576-586.

3 F. Zhou, X. L. Li, W. L. Wang, P. Zhu, J. Zhou, W. Y. He, M. Ding, F. Y. Xiong, X. N. Zheng, Z. Li, Y. L. Ni, X. H. Mu, L. Wen, T. Cheng, Y. Lan, W. P. Yuan, F. C. Tang and B. Liu, Nature, 2016, 533, 487-492.

4 N. Navin, J. Kendall, J. Troge, P. Andrews, L. Rodgers, J. McIndoo, K. Cook, A. Stepansky, D. Levy, D. Esposito, L. Muthuswamy, A. Krasnitz, W. R. McCombie, J. Hicks and M. Wigler, Nature, 2011, 472, 90-94.

5 D. J. Eastburn, A. Sciambi and A. R. Abate, Nucleic Acids Res., 2014, 42, e128.

6 D. K. Wood, D. M. Weingeist, S. N. Bhatia and B. P. Engelward, Proc. Natl. Acad. Sci. U. S. A., 2010, 107, 10008-10013.

7 Y. X. Zhao, F. Chen, Q. Li, L. H. Wang and C. H. Fan, Chem. Rev., 2015, 115, 12491-12545.

8 M. Ennen, C. Keime, D. Kobi, G. Mengus, D. Lipsker, C. Thibault-Carpentier and I. Davidson, Oncogene, 2015, 34, 3251-3263. 
9 Y. Zhu, Y. X. Zhang, W. W. Liu, Y. Ma, Q. Fang and B. Yao, Sci. Rep., 2015, 5, 09551.

10 I. C. Clark and A. R. Abate, Lab Chip, 2017, 17, 2032-2045.

11 Y. H. Zhang and H. R. Jiang, Anal. Chim. Acta, 2016, 914, 7-16.

12 D. J. Eastburn, A. Sciambi and A. R. Abate, Anal. Chem., 2013, 85, 8016-8021.

13 Z. Li, Y. Liu, Q. Q. Wei, Y. J. Liu, W. W. Liu, X. L. Zhang and Y. D. Yu, PLoS One, 2016, 11, e0153359.

14 I. K. Dimov, R. Lu, E. P. Lee, J. Seita, D. Sahoo, S. M. Park, I. L. Weissman and L. P. Lee, Nat. Commun., 2014, 5, 3451. 15 I. G. Wilson, Appl. Environ. Microbiol., 1997, 63, 3741-3751. 16 W. Abu al-Soud and P. Radstrom, J. Clin. Microbiol., 2001, 39, 485-493.

17 P. Radstrom, R. Knutsson, P. Wolffs, M. Lovenklev and C. Lofstrom, Mol. Biotechnol., 2004, 26, 133-146.

18 A. T. Hall, A. M. Zovanyi, D. R. Christensen, J. W. Koehler and T. D. Minogue, PLoS One, 2013, 8, e73845.

19 A. V. P. Le, D. X. Huang, T. Blick, E. W. Thompson and A. Dobrovic, Sci. Rep., 2015, 5, 12859.

20 D. A. David Svec, M. Pekny, R. Sjöback, M. Kubista and S. Anders, Front Oncol., 2013, 3, 274.
21 L. A. Torre, F. Bray, R. L. Siegel, J. Ferlay, J. Lortet-Tieulent and A. Jemal, Ca-Cancer J. Clin., 2015, 65, 87-108.

22 J. M. M. Walboomers, M. V. Jacobs, M. M. Manos, F. X. Bosch, J. A. Kummer, K. V. Shah, P. J. F. Snijders, J. Peto, C. Meijer and N. Munoz, J. Pathol., 1999, 189, 12-19.

23 K. Funakoshi, M. Bagheri, M. Zhou, R. Suzuki, H. Abe and H. Akashi, Sci. Rep., 2017, 7, 13202.

24 J. A. Walker, G. E. Kilroy, J. Xing, J. Shewale, S. K. Sinha and M. A. Batzer, Anal. Biochem., 2003, 315, 122-128.

25 H. A. Cubie, A. L. Seagar, E. McGoogan, J. Whitehead, A. Brass, M. J. Arends and M. W. Whitley, J. Clin. Pathol.: Clin. Mol. Pathol., 2001, 54, 24-29.

26 K. M. Ostrowska, A. Garcia, A. D. Meade, A. Malkin, I. Okewumi, J. J. O'Leary, C. Martin, H. J. Byrne and F. M. Lyng, Analyst, 2011, 136, 1365-1373.

27 J. J. Oleary, G. Browne, M. I. Johnson, R. J. Landers, M. Crowley, I. Healy, J. T. Street, A. M. Pollock, F. A. Lewis, A. Andrew, C. Cullinane, O. Mohamdee, W. F. Kealy, J. Hogan and C. T. Doyle, J. Clin. Pathol., 1994, 47, 933-938. 